\title{
Conductas fisioterapéuticas
}

en a.rehabilitación temprana del paciente con terapia
de soporte circulatorio
extracorpóreo (ECMO) Revisión integradora de la literatura Physiotherapeutic behaviors in the early rehabilitation of the patient with extracorporeal circu-

latory support therapy (ECMO): An integrative review of the literature

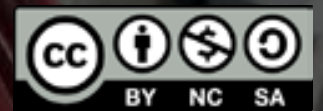
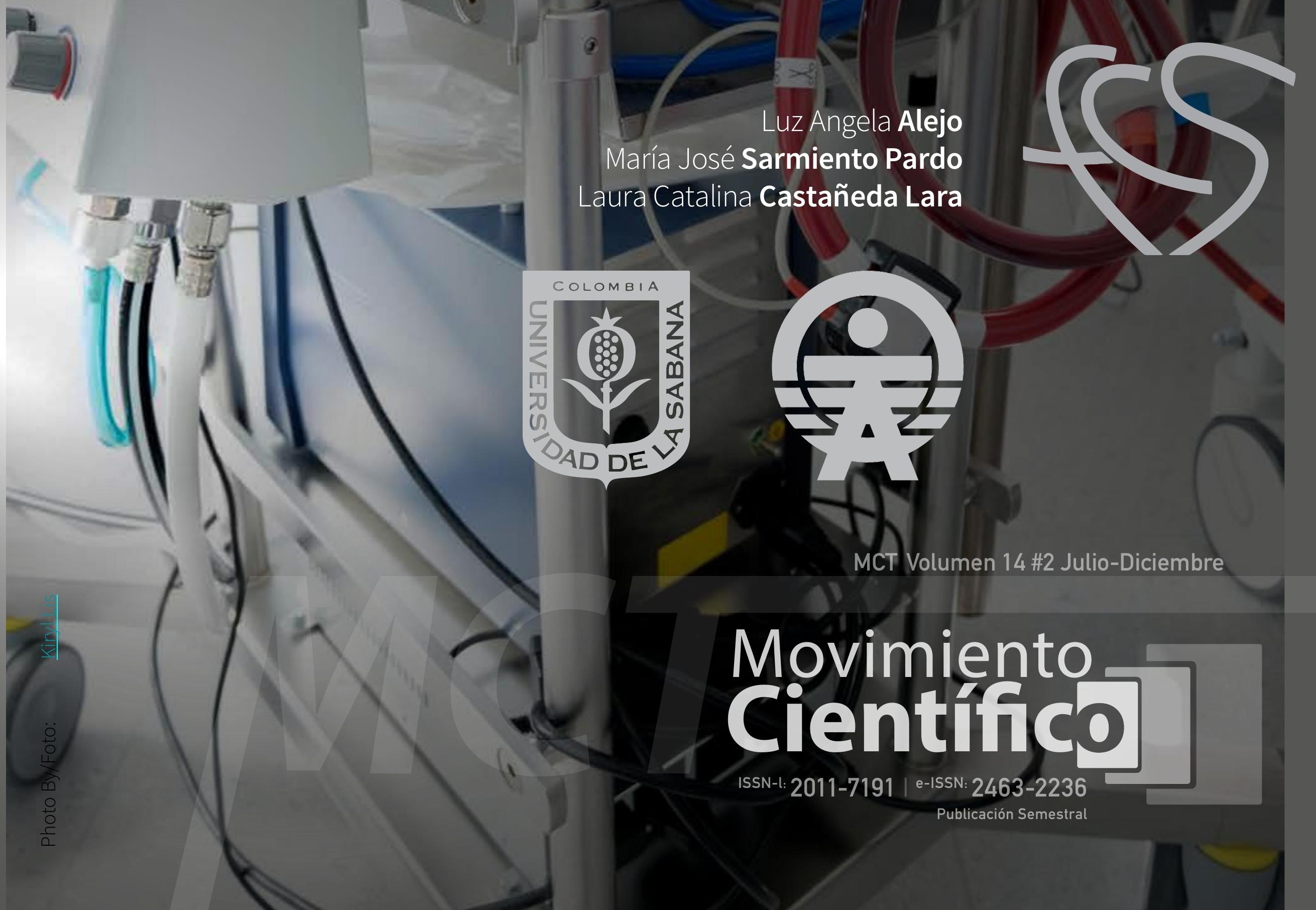


\section{Movimiento Cientifico}

ISSN-l: 2011-7191 | e-ISSN: 2463-2236 Publicación Semestral

ID: 2011-7197.mct.14201

Title:

Physiotherapeutic behaviors in the early rehabilitation of the patient with extracorporeal circulatory support therapy (ECMO)

Subtitle: An integrative review of the literature

Título: $\quad$ Conductas fisioterapéuticas en la rehabilitación temprana del paciente con terapia de soporte circulatorio extracorpóreo (ECMO)

Subtítulo: Revisión integradora de la literatura

Alt Title / Título alternativo:

[en]: $\quad$ Physiotherapeutic Behaviors in the recovery of the patient with extracorporeal circulatory support therapy (ECMO) an integrative review of the literature

[es]: $\quad$ Conductas fisioterapéuticas en la rehabilitación temprana del paciente con terapia de soporte circulatorio extracorpóreo (ECMO) Revision Integradora de la Literatura

Author (s) / Autor (es):

Alejo, Sarmiento Pardo \& Castañeda Lara

Keywords / Palabras Clave:

[en]: $\quad$ Ecmo, Physical Therapy Modalities, Mobilization, Earl.

[es]: $\quad$ Ecmo, Physical Therapy Modalities, Mobilization, Earl.

Submited: 2018-09-18

Acepted: 2021-04-07

\section{Resumen}

Introducción: La oxigenación con membrana extracorpórea (ECMO), es una técnica de asistencia circulatoria y/o respiratoria durante un período de días o meses, para lo cual, se somete al paciente a sedación de bloqueo neuromusculary analgesia asegurando vía aérea, por medio de un soporte ventilatorio.

Aunque, es una intervención potencial, puede llegar a afectar la calidad de vida por los tiempos prolongados de inmovilización.

Objetivo: Describir la evidencia disponible sobre las conductas fisioterapéuticas dirigidas a pacientes sometido a terapia de membrana extracorpórea y de esta manera demarcar la importancia del fisioterapeuta

especialista en cuidado crítico dentro del grupo transdisciplinar que genera atención en salud a este tipo de pacientes al interior de la UCI. Método: Se realizó una revisión integradora de la literatura, bajo la metodología de Ganong donde se analizaron datos relacionados con las conductas

fisioterapéuticas utilizadas en pacientes con esta técnica. Resultados: Se evidencia poca literatura científica que proporcione

altos niveles de evidencia y grado de recomendación que permitan analizar la importancia del trabajo fisioterapéutico en pacientes sometidos a esta terapia. Conclusión: La mayoría de los artículos

analizados sobre la terapia ECMO son estudios con un nivel de evidencia y grado de recomendación bajo que se enfocan en la participación del fisioterapeuta desde los dominios osteo y/o neuromuscular, dejando de lado dominios en que el especialista en cuidado crítico actúa, desde asegurar vía aérea hasta la optimización de la función multisistémica con el uso de dispositivos de asistencia como es el soporte ventilatorio.

\section{Abstract}

Introduction: Extracorporeal membrane oxygenation (ECMO) is a circulatory and/or respiratory support technique for a period

of day or months, for which the patient

is subjected to neuromuscular blockade sedation and analgesia ensuring airway, by means of ventilatory support. Although it is a potential intervention, it can affect the quality of life due to prolonged immobilization times. Objective: To describe the available evidence on the physiotherapeutic conducts directed

to patients summited to extracorporeal membrane therapy and in this way demarcate the importance of the physiotherapist specialist inside the ICU. Method: An

integrative review of the literature was carried out under the Ganong methodology where data related to the physiotherapeutic work in patients undergoing this therapy. Results: There is little scientific literature that provides high levels of evidence and degree of recommendation to analyze the importance of physiotherapeutic work in patients undergoing this therapy. Conclusion: Most

of the articles analyzed on ECMO therapy are studies with a low level of evidence and

degree of recommendation that focus on the participation of the physiotherapist from the osteo and/or neuromuscular domains,

leaving aside domains in which the critical care specialist acts, from securing the airways to the optimization of multisystemic function with the use of assistive devices such as ventilatory support.

\section{Citar como:}

Alejo, L. A., Sarmiento Pardo, M. J., \& Castañeda Lara, L. C. (2020). Conductas fisioterapéuticas en la rehabilitación temprana del paciente con terapia de soporte circulatorio extracorpóreo (ECMO): Revisión integradora de la literatura. Movimiento Científico , 14 (2), 61-69. Obtenido de: https:// revmovimientocientifico.ibero.edu.co/article/view/mct.14201

Luz Angela Alejo, msc Ft.

Source | Filiacion:

Universidad de la Sabana

BIO:

Fisioterapeuta. Mágister. Docente Universidad de la Sabana

City | Ciudad:

Bogotá DC [CO]

e-mail:

alejoluzangela@gmail.com
María José Sarmiento Pardo, Ft.sp

Source | Filiacion:

Hospital San Ignacio

BIO:

Fisioterapeuta. Especialista en Fisioterapia en cuidado crítico.

City | Ciudad:

Bogotá DC [Co]

e-mail:

majose303@gmail.com
Laura Catalina Castañeda Lara, Ft.sp

Source | Filiacion:

Fundación Abood Shaio

BIO:

Fisioterapeuta. Especialista en Fisioterapia en cuidado crítico.

City $\mid$ Ciudad:

Bogotá DC [CO]

e-mail:

catalina190411@gmail.com 


\title{
Conductas fisioterapéuticas en la rehabilitación
}

\author{
temprana del paciente \\ con terapia de soporte \\ circulatorio extracorpóreo

Revisión integradora de la literatura

Physiotherapeutic behaviors in the early rehabilitation of the patient with extracorporeal circulatory support therapy (ECMO): An integrative review of the literature

\section{Introducción}

La oxigenación con membrana extracorpórea (ECMO), es una técnica de soporte vital disponible por medio de un sistema de asistencia mecánica circulatoria (asistencia venoarterial V-A ECMO) y respiratoria (asistencia venovenosa V-V ECMO), capaz de proporcionar soporte cardíaco y/o pulmonar durante un periodo de días o meses para la insuficiencia cardíaca o respiratoria refractaria después del tratamiento convencional (Tramm, y otros, 2015). Para poder llevar a cabo esta técnica, es necesario someter al paciente en primera instancia a sedación de bloqueo neuromuscular y analgesia para lo cual se debe asegurar vía aérea, por medio de un soporte ventilatorio adicional.

Aunque la terapia ECMO, es una intervención potencial para disminuir la mortalidad de pacientes con cualquier tipo de enfermedad cardiopulmonar, puede provocar complicaciones tales como: sangrado, infección de la zona canulada, lesiones vasculares y déficits tanto nerviosos como sensitivos en las extremidades después de la canulación del vaso, ya sea femoral o arterial (Mateen, y otros, 2011), y así mismo, el tiempo de inmovilización prolongada en cama puede llegar a tener un impacto negativo disminuyendo la calidad de vida de la persona (Zangrillo, y otros, 2013). 
Paralograrlo escrito anteriormente, el fisiotera peuta especialista,

Históricamente se empezó a conocer sobre la terapia ECMO desde el año 1885 cuando Frey y Gruber desarrollaron el primer dispositivo capaz de oxigenar sangre extracorpóreamente. Más adelante John Gibbon vio morir a un joven por una embolia pulmonar y junto con su esposa empezaron a desarrollar la primera máquina corazón pulmón con soporte técnico; el 6 de mayo de 1953 realizan la primera cirugía a corazón abierto con circulación extracorpórea en Philadelphia (Mateen, y otros, 2011).

En los años 60 el doctor Theodor Kolobow y Col, desarrollaron el primer pulmón artificial (oxigenador) de membrana, sometiendo a su vez varios pacientes a este tipo de terapia prolongada sin obtener éxito alguno. Sin embargo, Robert H. Bartlett, quien es considerado el padre del ECMO, en 1975 realizó con éxito el primer ECMO neonatal a una paciente con falla respiratoria e hipertensión pulmonar secundario a una aspiración meconial (Díaz, Fajardo, \& Rufs, 2017). A su vez es el fundador de Elso (Extracorporeal Life Support Organization) una organización internacional sin ánimo de lucro con la base de datos más grande del mundo, que desarrolla guías clínicas para el manejo de paciente en ECMO ya sea neonatal, pediátrico y/o adulto (Extracorporeal Life Support Organization (ELSO), 2013).

Desde el año 2007, Colombia comenzó a aplicar esta técnica, la cual ha aumentado su aplicación en centros de cuarto nivel de atención, ya que puede sustituir hasta el cien por ciento de la función cardiaca y/o respiratoria, estabilizando la situación clínica del paciente, dando tiempo para reparar órganos afectados o para reemplazarlos con trasplantes u órganos artificiales

2010).A su vez los centros ECMO deben ser centros que se encuentren localizados en regiones geográficas donde puedan dar soporte al menos a 6 casos de ECMO por año (Extracorporeal Life Support Organization-ELSO, 2014).

El ECMO es un sistema complejo que requiere de un equipo transdisciplinario conformado por un médico que funcione como director del programa ECMO con la responsabilidad completa del centro. Junto a él se contará con equipo médico especializado, enfermeros, fisioterapeutas especializados en cuidado crítico, ingenieros biomédicos, nutricionistas, per fusionistas y psicólogos, entre otros (Extracorporeal Life Support Organization - ELSO, 2014). Cada uno de estos profesionales debe tener la experiencia necesaria en terapia intensiva y ser certificados en terapia ECMO.

El fisioterapeuta especialista en el área clínica enfocada al cuidado crítico como elemento primordial en este grupo médico que brinda atención al paciente sometido a terapia ECMO, tiene como objetivo estudiar, analizar evitar e intervenir las posibles alteraciones del movimiento corporal humano, a través del bienestar cinético y emocional.

El ejercicio profesional del especialista en fisioterapia en cuidado crítico, ha traspasado el dominio cardiovascular- pulmonar, a través del uso de técnicas de ahorro de energía para mantener la fuerza-resistencia diafragmática, el manejo de soporte ventilatorio y la permeabilidad de la vía aérea, en busca de disminuir las complicaciones durante y después de la terapia de soporte. Hoy en día, dicho especialista, impacta todos los dominios o ámbitos sistémicos, propiciando la activación muscular precoz o anticipada, como un pilar de intervención fisioterapéutica.

\section{Materiales y métodos}

Se llevó a cabo una revisión integradora de la literatura, bajo la metodología de búsqueda integradora de Ganong, la cual plantea un número de etapas para la investigación (Gráfico 1).

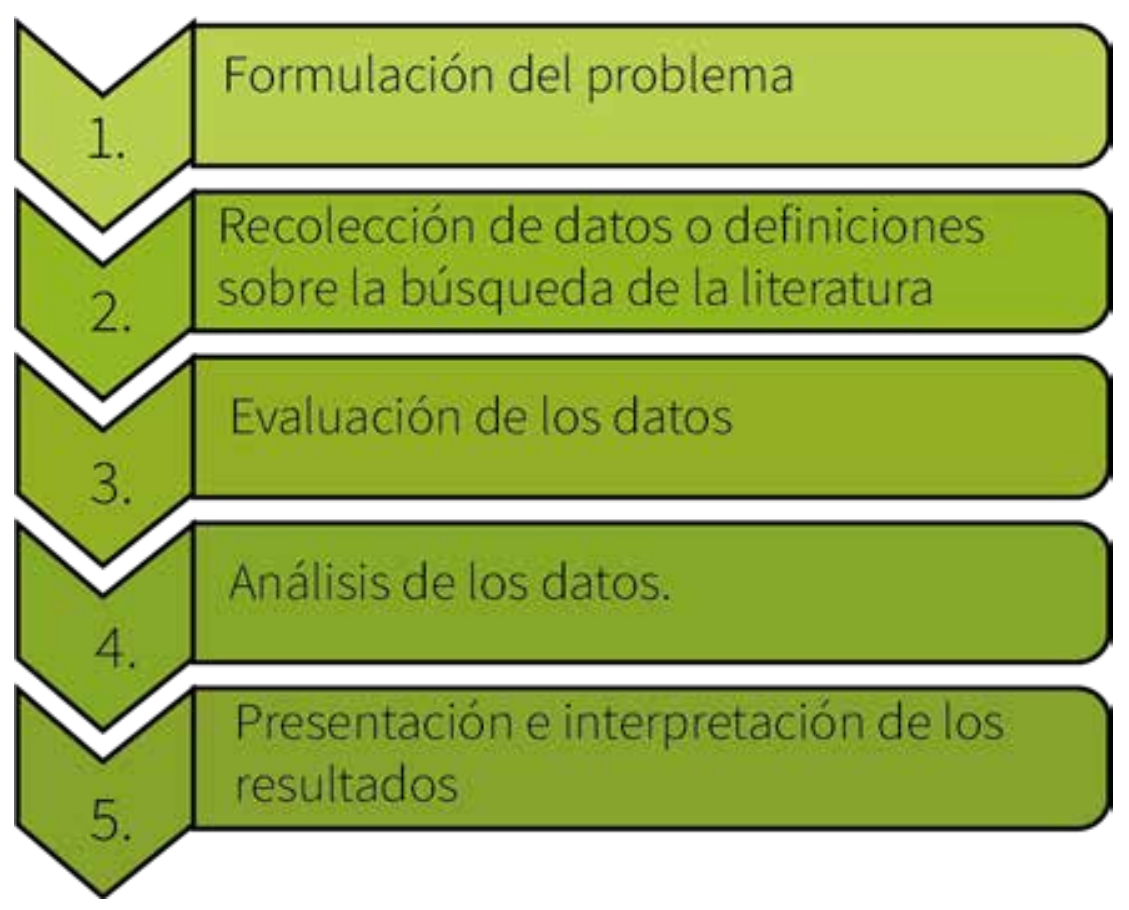

Gráfico 1 Búsqueda integradora de Ganong (Elaboración propia).

De acuerdo con lo anterior se utilizan bases de datos como Science Direct, Clinical Key, ProQuest y Pubmed bajo las siguientes fórmulas de búsqueda (Gráfico 2). 


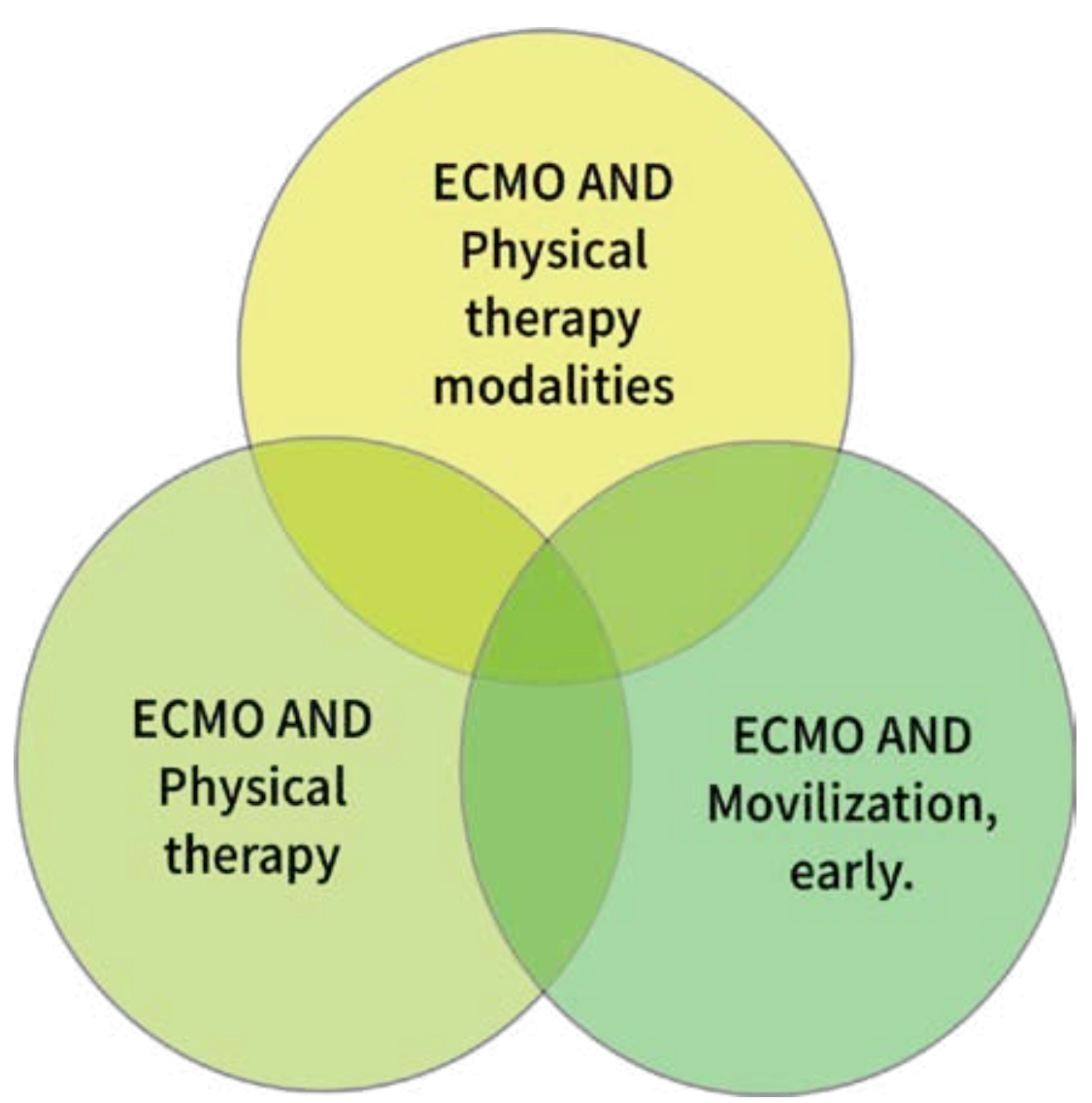

Gráfico 2 Fórmulas de búsqueda (Elaboración propia).

Como criterios de Inclusión se tuvieron en cuenta revisiones de la literatura, estudios de caso y controles, estudios experimentales, revisiones sistemáticas y guías clínicas basadas en la evidencia en el paciente adulto de cuidado intensivo con terapia de soporte circulatorio extracorpóreo (ECMO) por disfunción primaria cardiovascular-pulmonar; en idioma inglés, español y portugués, con un rango de publicación entre el año 2000 y 2018.

Como criterios de exclusión: estudios que no tengan relación con las palabras clave, terapia de soporte circulatorio extracorpóreo (ECMO) en pacientes neonatales, pediátricos o con disfunción primaria en los dominios osteomuscular, neuromuscular y tegumentario en etapa aguda en $\mathrm{UCl}$.

Para la evaluación, organización y análisis de los resultados se elaboraron dos matrices, una bibliométrica y otra de análisis de artículos, de acuerdo con lo anterior, se procedió a su análisis de acuerdo con las fechas, idiomas, metodología, lugares de procedencia, niveles de evidencia y contenido buscando en estas similitudes, complementos y diferencias de los temas relativos al fenómeno estudiado.

\section{Resultados}

A continuación, se exponen los resultados más importantes encontrados en el desarrollo de esta investigación.

En el Gráfico 3, recolección de evidencia científica, se ilustra el proceso de recolección de artículos científicos con respecto a las fórmulas de búsquedas utilizadas. Se realizó bajo la combinación de ECMO con modalidades fisioterapéuticas, fisioterapia, movilización y temprana. Se recopilaron una cantidad importante de artículos con palabras clave, al ahondar en sus resúmenes se lograron concretar 50 artículos sobre los cuales se realizó el análisis integrador.

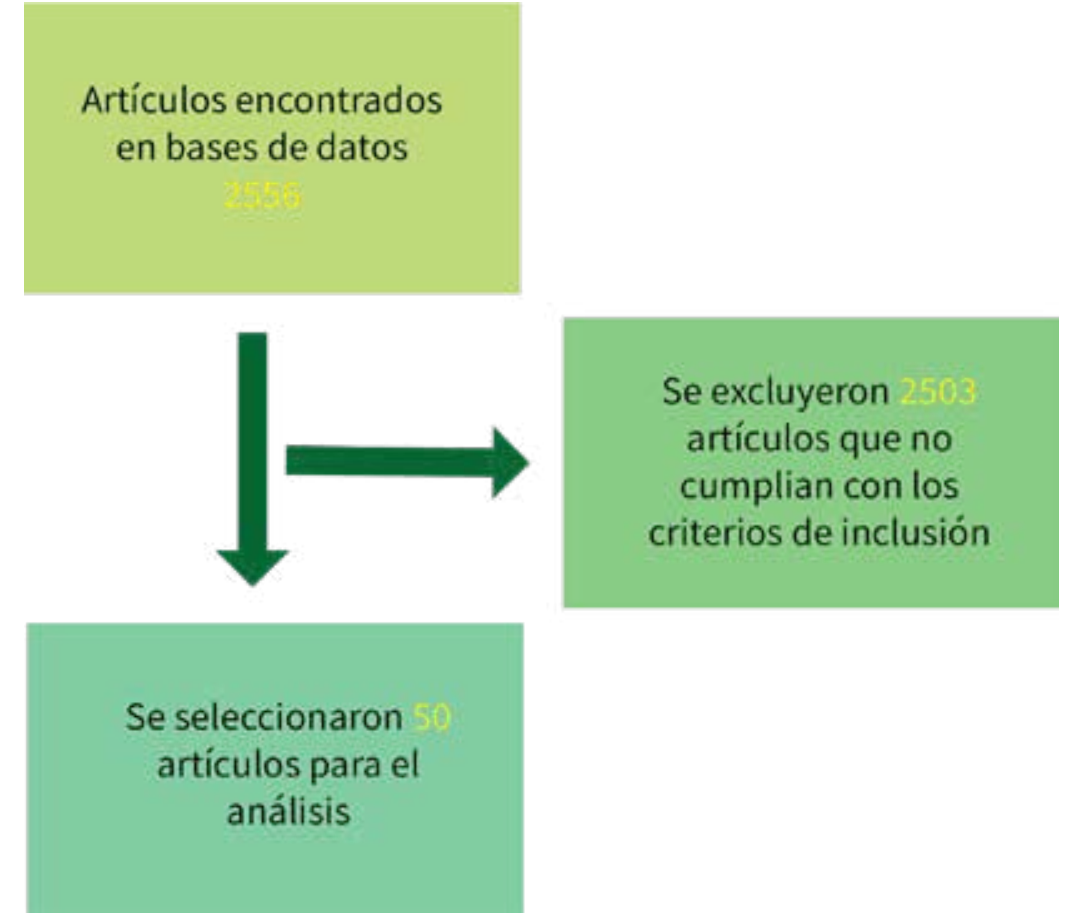

Gráfico 3 Recolección de evidencia científica (Elaboración propia).

Al seleccionar la evidencia científica se comenzaron a realizar los análisis respectivos de acuerdo con las matrices elaboradas por las autoras, teniendo en cuenta aspectos como el área geográfica, lugar y año de publicación, tipo de ECMO, conducta fisioterapéutica utilizada y niveles de evidencia y grados de recomendación.

En el Gráfico 4, a nivel mundial se evidenció que la zona geográfica en donde más se realiza investigación con respecto a la fisioterapia en ECMO es en América con un 68\% de la evidencia encontrada, seguido por Europa con 24\%, Asia con un 6\% y Oceanía con un $2 \%$.

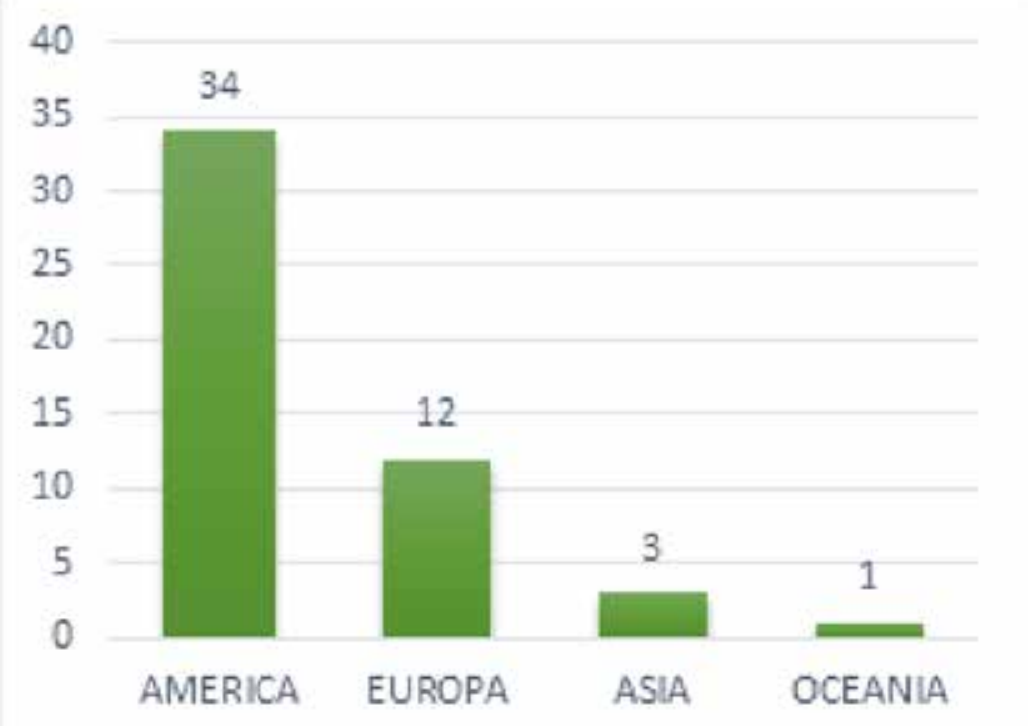

Gráfico 4 Número de artículos por área geográfica (Elaboración propia).

Es importante para este estudio conocer sobre el estado de actualización de las investigaciones sobre la fisioterapia en la terapia ECMO, según el análisis de la evidencia científica, los artículos seleccionados se publicaron en un rango de tiempo desde el 2008 al 2018, de los cuales 41 estudios fueron publicados en idioma inglés y 9 en español; aunque los criterios de búsqueda incluían estudios en portugués en la selección de artículos ninguno estaba publicado en este idioma. En la Tabla 1. se puede observar un incremento en el interés sobre esta temática en el año 2014, 2015 y 2017.

En cuanto al tipo de ECMO, que se observa en la Gráfico 5. Se encontró que 27 artículos estaban enfocados a terapia ECMO venovenoso, 9 artículos para ECMO veno- arterial, 1 articulo para ECMO veno- arterial- venoso y 13 artículos que se enfocaban en ambas técnicas. 


\section{Conductas fisioterapéuticas en la rehabilitación temprana del paciente con terapia de soporte circulatorio extracorpóreo (ECMO)}

\section{Revisión integradora de la literatura}

Se tuvo en cuenta para este estudio las conductas fisioterapéuticas utilizadas en los artículos, para así tener una visión del manejo global del fisioterapeuta en pacientes con este tipo de tratamiento. En el Gráfico 6, se puede evidenciar que según los artículos seleccionados se han realizado más investigaciones con respecto al manejo fisioterapéutico en dispositivos de asistencia y ventilación mecánica, no sin dejar a un lado la importancia del ejercicio físico y las demás conductas fisioterapéuticas.

Tabla 1. Número de artículos por año.

\begin{tabular}{|c|c|}
\hline Año & \#Artículos \\
\hline 2008 & 1 \\
\hline 2009 & 2 \\
\hline 2010 & 1 \\
\hline 2011 & 1 \\
\hline 2012 & 3 \\
\hline 2013 & 4 \\
\hline 2014 & 8 \\
\hline 2015 & 14 \\
\hline 2016 & 4 \\
\hline 2017 & 13 \\
\hline 2018 & 2 \\
\hline
\end{tabular}

Fuente: Elaboración propia.

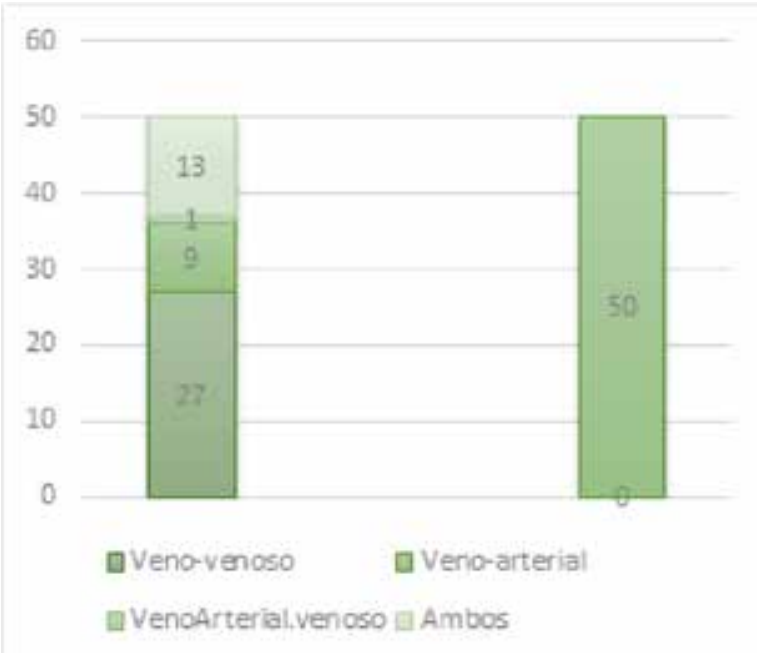

Gráfico 5 Número de artículos por tipo de terapia ECMO (Elaboración propia).

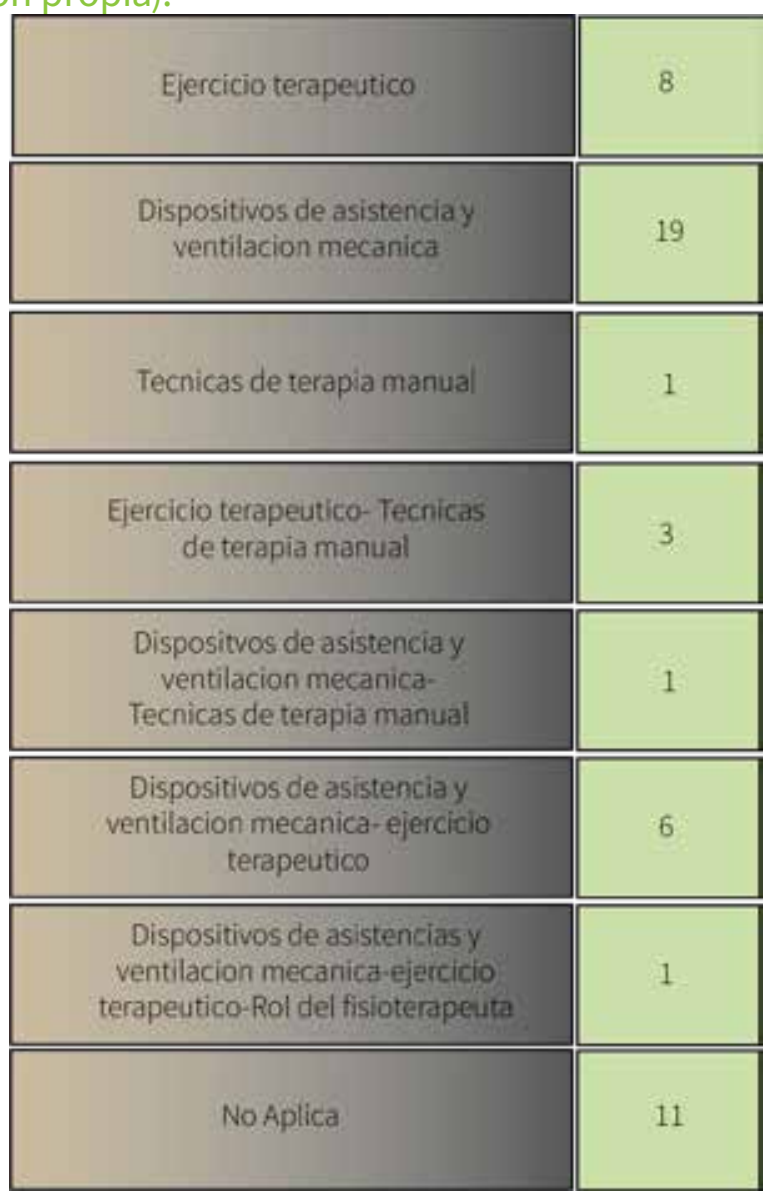

Gráfico 6 Número de artículos por conducta fisioterapéutica utilizada en pacientes con terapia ECMO (Elaboración propia).
Cada artículo seleccionado se clasificó de acuerdo con el tipo de estudio, nivel de evidencia y grados de recomendación según la escala de Oxford. En cuanto al tipo de estudio se encontraron 24 artículos de casos y cohortes, 15 revisiones de literatura, 3 revisiones sistemáticas, 7 estudios observacionales y un metaanálisis los cuales se pueden observar en la Tabla de Numero De Artículos Por tipo de Estudio, Tabla 2.

Los grados de evidencia y recomendación según Oxford, se caracterizan por valorar la evidencia según la temática o escenario clínico y el tipo de estudio que involucra al problema clínico en cuestión, a su vez gradúa la evidencia de acuerdo con el mejor diseño para cada escenario clínico (Manterola, Zavando, \& MINCIR, 2009).

Tabla 2. Número de artículos por tipo de estudio.

\begin{tabular}{|l|c|}
\hline \multicolumn{1}{|c|}{ Tipo de estudios } & Número \\
\hline Casos y Cohortes retrospectivos & 24 \\
\hline Revisión de literatura & 15 \\
\hline Estudios Observacionales & 7 \\
\hline Metaanálisis & 1 \\
\hline Revisión Sistemática & 3 \\
\hline
\end{tabular}

Fuente: Elaboración propia.

De acuerdo con el Gráfico 7, se clasificaron los artículos evidenciando que en la literatura aún faltan estudios de investigación que proporcionen altos grados de evidencia y recomendación los cuales permitan evidenciar la importancia del trabajo fisioterapéutico en pacientes con terapia ECMO.

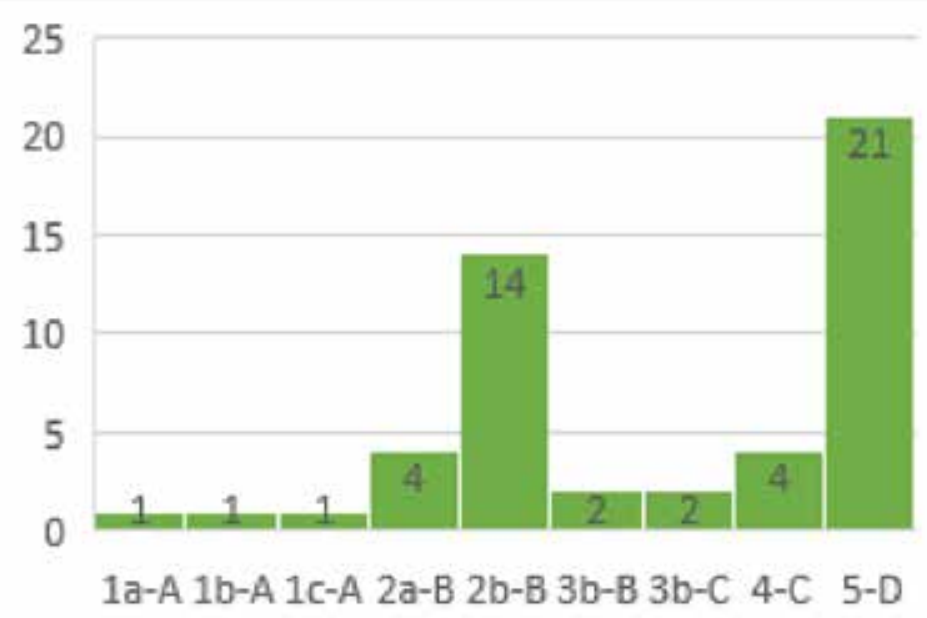

Gráfico 7 Número de artículos por niveles de evidencia y grado de recomendación (Elaboración propia).

\section{Discusión}

El fisioterapeuta en su quehacer profesional trabaja con diferentes conductas las cuales hacen referencia a la variedad de estrategias que el profesional utiliza para intervenir y generar efectos sobre deficiencias tanto estructurales, y/o funcionales, así como las limitaciones y restricciones que puede llegar a presentar una persona con una condición de salud en particular, según la Ley 528 de 1999 (Congreso de Colombia, 1999). De acuerdo con lo anterior, para este estudio se tuvo en cuenta tres conductas principales del quehacer fisioterapéutico: Ejercicio terapéutico, técnicas de terapia manual y técnicas de permeabilización de la vía aérea.

Según los resultados obtenidos de la matriz de artículos, de un total de 50 artículos solo 8 hablan específicamente del uso de modalidades de intervención enfocadas a ejercicios de flexibilidad, 
acondicionamiento y reacondicionamiento aeróbico, así como entrenamiento de la fuerza y resistencia muscular.

Polastri et al. en su estudio Physiotherapy for Patients on Awake Extracorporeal Membrane Oxygenation: A Systematic Review, (2016); menciona que la participación de pacientes con terapia Ecmo en terapia física es una opción viable para evitar des acondicionamiento físico por inmovilidad prolongada, este estudio propone que ejercicios enfocados en entrenamiento para deambulación, patrones funcionales de movimiento para extremidades y entrenamiento de fuerza progresiva, permite la rehabilitación temprana de este tipo de paciente, pues evita la inmovilización prolongada en cama y complicaciones a nivel respiratorio como dependencia al soporte ventilatorio, mencionando que el paciente debe estar despierto y consciente, promoviendo la participación de la terapia física activa mientras esté en la terapia de soporte extracorpóreo, siendo una opción viable para su rehabilitación siempre y cuando se garantice la cooperación entre todos los profesionales involucrados para garantizar la seguridad del paciente. Sin embargo, Schweickerty Kress (2011), mencionan que la terapia física enfocada a posicionamiento en cama y la movilización pasiva evita el desplazamiento de las cánulas de la terapia ECMO disminuyendo complicaciones.

No obstante, las intervenciones enfocadas a posicionamiento en cama y movilización temprana según APTA, 2014; hacen parte de las técnicas de terapia manual definidas como habilidades para realizar movimientos pasivos de articulaciones y tejidos blandos, para mejorar la extensibilidad tisular, incrementar rangos de movimiento, modulación del dolor e inflamación, para mantener y mejorar el desempeño físico y así mismo, prevenir o disminuir deficiencias tanto estructurales como funcionales (American Physical Therapy Association, 2014).

Es importante tener en cuenta que la terapia de soporte extracorpóreo maneja tres tipos de canulación: una central (ECMO VENO-ARTERIAL), una periférica (ECMO VENO-VENOSO y mixta (ECMO VENOVENOSO-ARTERIAL) que combina canulación central y periférica. Perme y otros (2013) publicaron un estudio observacional , en el cual examinó la incidencia de efectos adversos relacionados con el catéter femoral durante las intervenciones de terapia física para pacientes adultos en una $\mathrm{UCl}$ cardiovascular, describiendo que la movilidad temprana en la UCl para pacientes hemodinámica mente estables y que cumplieran con criterios de movilización con catéteres arteriales y venosos femorales, eran importantes para minimizar el deterioro funcional; pues estas intervenciones estaban enfocadas a transferencias de peso, movilizaciones pasivas y cambios de posición de manera progresiva, de igual manera como se trabaja en un paciente en $\mathrm{UCl}$, exceptuando la movilización de las extremidades donde se encuentre ubicado la canulación del equipo extracorpóreo.

Así mismo, según el análisis de la evidencia encontrada, la conducta por si sola de terapia manual no es enfocada solo para el paciente con terapia ECMO, sino también en pacientes con otro tipo de alteraciones dependiendo de la fase en que se encuentre.

Kate Hayes y otros en 2016, realizó un estudio retrospectivo donde describió la función física, las complicaciones de las extremidades inferiores y la calidad de vida relacionada con la salud (CVRS) en los tres meses después de la terapia ECMO antes o después del trasplante cardíaco. Este es el primer estudio para describir la función física en pacientes que reciben ECMO pre o post cirugía cardiaca, la mayoría de pacientes demostraron debilidad muscular severa adquirida en UCl por inmovilidad prolongada, asociándose esta con la disminución de la síntesis de proteína y atrofia muscular, mientras que la enfermedad crítica se asocia con una aumento del estado catabólico con regulación positiva de mediadores pro inflamatorios, así como, cambios en la composición muscular que conducen a la debilidad (Hayes, y otros, 2016).

Sin embargo, Hayes y otros (2016) describen que el enfoque de la movilización temprana en el contexto de la UCl sea igual tanto para el paciente en terapia ECMO como para el paciente en vigilancia por trastornos estructurales o funcionales de cualquier origen, siendo esta una opción terapéutica posible para mejorar la fuerza muscular, la función física y la calidad de vida, disminuyendo así comorbilidades y aumentando niveles de supervivencia. Es por esto, que los ejercicios de fortalecimiento de las extremidades favoreciendo la deambulación en pacientes con ECMO puede ser una intervención efectiva para reducir la incidencia de la debilidad muscular adquirida en UCl. Sin embargo, existen barreras para la deambulación mientras se está en ECMO, por lo que se trabajan técnicas de terapia manual que se basan en ahorro de energía y disminución de consumo metabólico.

En este tipo de paciente también se tiene en cuenta que, para poder llevar a cabo esta técnica, es necesario someter al paciente en primera instancia a sedación de bloqueo neuromuscular y analgesia para lo cual se debe asegurar vía aérea, por medio de un soporte ventilatorio adicional. Este tipo de asistencia se incluiría según el que hacer fisioterapéutico en técnicas de permeabilización de la vía aérea, desde el uso de soporte de asistencia ventilatoria, en los cuales el fisioterapeuta especialista en cuidado crítico puede prescribir, aplicar o modificar parámetros para mejorar deficiencias funcionales atribuidas principalmente por deficiencias estructurales ya sea por injuria cardiaca, pulmonar o de interacción multisistémica como la interacción corazón- pulmón (Hayes Jr., y otros, 2013).

Los estudios sobre aplicación de la terapia de ECMO tienen un aumento de estudio y publicación según el análisis de la evidencia científica seleccionada en los años 2014, 2015 y 2017, que según la organización mundial de la salud concuerda con el aumento y la prevalencia de enfermedades de tipo respiratorio como la neumonía y complicaciones de esta como el síndrome de dificultad respiratoria aguda (SDRA) por virus como el H1N1 o adenovirus. La pandemia de virus H1N1 en todo el mundo, afectó en mayor proporción a población joven que adulta mayor, en contraste con los patrones típicos de influenza estacional. Por lo que una proporción significativa de estos pacientes más jóvenes con H1N1 no mejoraron con estrategias de ventilación mecánica convencional, lo que provocó la rápida expansión de la utilización de ECMO veno-venoso en unidades de cuidados intensivos a nivel mundial. Estudios retrospectivos sobre la experiencia de la influenza H1N1, favorecen el uso de ECMO en pacientes con síndrome de dificultad respiratoria aguda. Sin embargo, la eficacia del ECMO veno-venoso en relación con la resolución de H1N1 y el SDRA sigue siendo incierto ya que los pacientes con este mismo diagnóstico pero que no fueron manejados con ECMO tuvieron resultados similares en algunos estudios (Zangrillo, y otros, 2013); (Orrego \& Díaz, 2017).

EncuantoalaECMOveno-arterial,Orregoy Díaz (2017)mencionan en su estudio Reanimación Cardiopulmonar Extracorpórea: La última Frontera, que "el uso del ECMO veno-arterial surge como una terapia efectiva al restaurar el flujo sanguíneo en aquellos pacientes en paro cardiorrespiratorio que no responden a las maniobras convencionales óptimas de reanimación cardiopulmonar. Permite mejorar la protección cerebral, manejarel síndrome post-reperfusión, disminuyendo la mortalidad postparo cardiorrespiratorio y como un puente para toma de decisiones al otorgar tiempo para realizar trasplantes". 


\section{Conductas fisioterapéuticas en la rehabilitación temprana del paciente con terapia de soporte circulatorio extracorpóreo (ECMO)}

\section{Revisión integradora de la literatura}

Autores como García Asenjo y Eiguren Goitiz (2017), mencionan que este tipo de asistencia mecánica puede utilizarse en el manejo del shock cardiogénico refractario, asocia una mortalidad precoz elevada, pero tras superar la fase hospitalaria la supervivencia de los pacientes es buena. Sin embargo, mencionan en su estudio que los artículos disponibles en la literatura están principalmente en la forma de informes de casos o series de caso de dos o tres pacientes, así como revisiones de literatura, lo que genera una escasez de datos sobre este tema a nivel de evidencia y grados de recomendación lo que concuerda con los resultados planteados de la base de artículos analizados en la cual solo se analizaron 9 artículos sobre ECMO venoarterial.

En cuanto a la categoría de técnicas de higiene de la vía aérea, APTA, 2014 define esta categoría como el grupo de actividades fisioterapéuticas destinadas a controlar o prevenir las consecuencias de las alteraciones del transporte mucociliar o la incapacidad para proteger la vía aérea seleccionando, prescribiendo e implementando, oxígeno suplementario y posicionamiento para garantizar la adecuada relación ventilación - perfusión, farmacoterapia y estrategias respiratorias manuales o mecánicas (Soporte ventilatorio) para reducir factores de riesgo multisistémicos y/o complicaciones (American Physical Therapy Association, 2014).

En la evidencia científica analizada se observó que, aunque en la mayoría de los artículos se encuentra en esta categoría y que mencionan la prescripción del soporte ventilatorio a volúmenes bajos, no mencionan el papel del fisioterapeuta en la prescripción y manejo del soporte ventilatorio ni en el manejo de la bomba centrífuga de la máquina extracorpórea encargada del intercambio gaseoso.

Solo uno de los 50 artículos analizados integra el papel del fisioterapeuta, Kate Hayes y otros (2016), describieron que la inmovilidad prolongada se asocia con la disminución de la síntesis de proteína muscular y la atrofia muscular, mientras que la enfermedad crítica se asocia con el aumento del estado catabólico con regulación positiva demediadores pro inflamatorios, que conducen a la debilidad muscular, por lo que la rehabilitación activa incluyendo ejercicio de fortalecimiento para las extremidades superiores e inferiores y deambulación mientras está en ECMO puede ser una intervención efectiva para reducir la debilidad observada y que es adquirida en cuidados intensivos. Así mismo, con la rehabilitación de fisioterapia después del alta de la UCl, los pacientes que analizaron en su estudio mostraron mejoras en la fuerza musculary la movilidad generalizada. Sin embargo, según este estudio la deambulación mientras está en ECMO, presenta varias barreras que incluyen la canulación femoral, sedación y ventilación mecánica. Este estudio si bien es el único en la evidencia analizada que responde al papel del fisioterapeuta, no presenta de manera integral el que hacer de esta profesión.

\section{CONCLUSIONES}

La mayoría de los artículos analizados sobre la terapia ECMO son estudios de casos y controles o revisiones de literatura, lo que genera un nivel de evidencia y recomendación baja para este tipo de terapia. Adicionalmente, solo se enfocan en la participación del fisioterapeuta desde el ámbito osteo y/o neuromuscular, lo que deja de lado la participación profesional integral del fisioterapeuta especialista en cuidado crítico, el cual inicia desde el manejo de la vía aérea hasta la optimización de la función multisistémica con el uso de dispositivos de asistencia como es el soporte ventilatorio.

En Colombia según el análisis de la matriz de artículos, solo se evidencian 3 estudios publicados sobre la terapia ECMO ya sea veno-venoso o veno-arterial y que cuentan con niveles de evidencia y recomendación baja, a su vez las intervenciones desde el punto de vista fisioterapéutico solo tienen en cuenta las conductas de técnicas mecánicas por medio de movilización temprana y ejercicio terapéutico, no incluyen la acción del fisioterapeuta especialista desde dispositivos de soporte ventilatorio que sustenta el que hacer desdeel dominio cardiovascular-pulmonar con el manejo del soporte ventilatorio y la programación de este si no también con técnicas de higiene de la vía aérea. Dando respuesta al perfil mencionado por la ley 528 de 1999 que responde a la fisioterapia como una profesión integral que evalúa, diagnostica e interviene cualquiera de los dominios ya sea cardiovascular-pulmonar, osteomuscular, neuromuscular e integumentario.

Es importante seguir en la búsqueda de información sobre este tema tan relevante y actual y seguir en la construcción del perfil profesional del especialista en fisioterapia en cuidado crítico.

\section{REFERENCIAS}

Abrams, D., Javidfar, J., Farrand, E., Mongero, L. B., Agerstrand, C. L., Ryan, P., . Brodie, D. (27 de Febrero de 2014). Early mobilization of patients receiving extracorporeal membrane oxygenation: a retrospective cohort study. Critical Care, 18(1), R38. doi:10.1186/cc13746

American Physical Therapy Association. (2014). APTA Guide to Physical Therapist Practice. Obtenido de https://guide.apta.org/

Congreso de Colombia. (1999). Ley 528 de 1999. Obtenido de https://www. mineducacion.gov.co/1621/articles-105013 archivo pdf.pdf

Díaz, R., Fajardo, C., \& Rufs, J. (2017). Historia del ECMO (Oxigenación por membrana extracorpórea o soporte vital extracorpóreo). Revista Médica Clínica Las Condes, 28(5), 796-802. doi:https://doi.org/10.1016/j. rmclc.2017.10.004

Extracorporeal Life Support Organization - ELSO. (Marzo de 2014). Guías ELSO para Centros ECMO. ELSO. Obtenido de https://www.elso.org/Portals/0/ Files/Guideline/ELSO\%20 Guias\%20para\%20Centros\%20de\%20ECM0\%20 Version\%201.8.pdf

Extracorporeal Life Support Organization (ELSO). (Diciembre de 2013). Guías para la insuficiencia Cardíaca en Adultos. Obtenido de https://www.elso. org/Portals/O/Files/Guideline/ELSO\%20Guias\%20para\%20Insuficiencia\%20 Cardiaca\%20del\%20Adulto\%201.3.pdf

García Asenjo, M., \& Eiguren Goitiz, K. (2017). Soporte vital extracorpóreo. Oxigenación por membrana extracorpórea. ECMO. Revista Española de Perfusión(62), 5-26. Obtenido de https://www.aep.es/revistaarticulo/24/62 2.pdf

Hayes Jr., D., Galantowicz, M., Yates, A. R., Preston, T. J., Mansour, H. M., \& McConnell, P. I. (2013). Venovenous ECMO as a bridge to lung transplant and a protective strategy for subsequent primary graft dysfunction. Journal of Artificial Organs, 16(3), 382-385. doi:10.1007/s10047-013-0699-Z

Hayes, K., Holland, A. E., Pellegrino, V. A., Leet, A. S., Fuller, L. M., \& Hodgson, C. L. (2016). Physical function after extracorporeal membrane oxygenation in patients pre or post heart transplantation - An observational study. Heart \& Lung: The Journal of Acute and Critical Care, 45(6), 525-531. doi:10.1016/j. hrtling.2016.07.007

Hsu, P.-S., Chen, J.-L., Hong, G.-J., Tsai, Y.-T., Lin, C.-Y., Lee, C.-Y., . . Tsai, C.-S. (2010) Extracorporeal membrane oxygenation for refractory cardiogenic shock after cardiac surgery: predictors of early mortality and outcome from 51 adult patients. European Journal of Cardio-Thoracic Surgery, 37(2), 328333. doi:10.1016/j.ejcts.2009.07.033

Manterola, C., Zavando, D., \& MINCIR, G. (Diciembre de 2009). Cómo interpretar los "Niveles de Evidencia" en los diferentes escenarios clínicos. Revista chilena de cirugía, 61(6), 582-595. doi:http://dx.doi.org/10.4067/S071840262009000600017 
Mateen, F. J., Muralidharan, R., Shinohara, R. T., Parisi, J. E., Schears, G. J., \& Wijdicks, E. F. (2011). Neurological injury in adults treated with extracorporeal membrane oxygenation. Archives of neurology, 68(12), 15431549. doi:10.1001/archneurol.2011.209

Orrego, R., \& Díaz, R. (2017). Reanimación Cardiopulmonar Extracorpórea: La Última Frontera. Revista Médica Clínica Las Condes, 28(2), 239-247. doi:https://doi.org/10.1016/j.rmclc.2017.04.011

Perme, C., \& Chandrashekar, R. (2009). Early Mobility and Walking Program for Patients in Intensive Care Units: Creating a Standard of Care. American Journal Of Critical Care, 18(3), 212-221. doi:10.4037/ajcc2009598

Perme, C., Nalty, T., Winkelman, C., Kenji Nawa, R., \& Masud, F. (Junio de 2013). Safety and Efficacy of Mobility Interventions in Patients with Femoral Catheters in the ICU: A Prospective Observational Study. Cardiopulmonary Physical Therapy Journal, 24(2), 12-17. Obtenido de https://www.ncbi.nlm. nih.gov/pmc/articles/PMC3691704/

Polastri, M., Loforte, A., Dell'Amore, A., \& Nava, S. (2016). Physiotherapy for Patients on Awake Extracorporeal Membrane Oxygenation: A Systematic Review. Physiotherapy Research International, 21(4), 203-209. doi:10.1002/pri.1644

Schweickert, W. D., \& Kress, J. P. (Diciembre de 2011). Implementing early mobilization interventions in mechanically ventilated patients in the ICU. Chest, 140(6), 1612-1617. doi:10.1378/chest.10-2829

Tramm, R., llic, D., Davies, A. R., Pellegrino, V. A., Romero, L., \& Hodgson, C. (22 de Enero de 2015). Extracorporeal membrane oxygenation for critically ill adults. Cochrane Database of Systematic Reviews, 1(1) doi:10.1002/14651858.CD010381.pub2

Zangrillo, A., Biondi-Zoccai, G., Landoni, G., Frati, G., Patroniti, N., Pesenti, A. \& Pappalardo, F. (13 de Febrero de 2013). Extracorporeal membrane oxygenation (ECMO) in patients with $\mathrm{H} 1 \mathrm{~N} 1$ influenza infection: a systematic review and meta-analysis including 8 studies and 266 patients receiving ECMO. Critical Care, 17(1), R30. doi:10.1186/cc12512 